\title{
Bone Augmentation with Bovine Bone Xenograft: 5 Years Result of Retrospective Cohort Study
}

\section{Nasim Mahjoub Pour, Hugo De Bruyn}

Department of Oral Health Sciences, Ghent University, Belgium

\begin{abstract}
Aim: The aim of this study is to investigate the crestal bone loss around the implants following guided bone regeneration in dehiscence and fenestrations and its effect on peri-implant health after a period of five years.

Materials and Methods: A total of 41 consecutively treated patients who had received bovine particulate bone augmentation Endobon ${ }^{\circledR}$ (Biomet 3i) were evaluated in a single center. From this cohort, 13 patients were enrolled in the study and the others were drop-outs.

17 implants were installed in the patients. The patients were contacted 5 years after implant loading. All patients had undergone periapical radiographs and orthopantogram immediately after crown installation T0 and then 5 years after placing the implants $\mathrm{T} 1$. Digital and analog radiographs were evaluated by one clinician from the patient charts after the crown installation. The periodontal chart was taken, pocket depth, bleeding on probing and plaque amount was scrutinized around each implant. Statistical analysis was performed using SPSS software

Results: All implants survived after 5 years of loading. Significant bone loss was revealed between the baseline and the follow-up time at the implant sites. The overall mean bone loss at the implant site was 0.69 (SD: 0.73). On the mesial side for all but two implants $(0.03$ and 0.04$)$ the evolution was toward bone loss. On the distal side for all but three implants $(0.01,0.02,0.02)$ the evolution was toward loss of bone. No difference in the bone loss was seen between mesial and distal sites.

$87.5 \%$ of the implant sites with bone augmentation showed bleeding on probing. The plaque was seen in $31.3 \%$ of the implant sites Conclusion: Bone augmentation with bovine bone hydroxyapatite in one stage implant surgery remained stable during the 5 years follow-up around the minimally rough implants used in this study. However, the amount of bone loss and mucositis at the implant sites with GBR remained significantly high.
\end{abstract}

\section{Key Words: Xenograft, Guided bone regeneration, Bone augmentation, Oral implants, Peri-implant health}

\section{Introduction}

Implant dentistry has undergone a revolutionary change during the last 20 years. However, clinicians still face difficulties to install implants in the narrow alveolar ridges.

Following a tooth extraction, the alveolar bone undergoes natural remodeling. This remodeling causes a serious dimensional alteration in the bone construction of the alveolar ridge which can lead to unfavorable aesthetic and functional results in the following prosthodontic treatment. In a recent systematic review, the auteur presents a mean reduction in the width of the alveolar ridge of about $3.87 \mathrm{~mm}$ [1]. It has been shown that the proportion of the dimensional changes is greater in the horizontal plane rather than in the vertical plane. These structural changes happen to be more noticeable at the facial aspect of an extraction site more than the lingual or palatal side [2].

In order to minimize the ridge resorption, different techniques have been utilized from which Guided Bone Regeneration (GBR) is the most popular. Guided regeneration was initially introduced in dentistry by Nyman and Karring. By using a barrier membrane to exclude undesired cells, they succeeded to partially regenerate lost periodontal tissue in periodontally affected teeth [3]. This idea was later on implemented into the other aspects of guided regeneration like guided bone regeneration or GBR. The biological rationale for GBR was to prevent ingrowth of non-osteogenic cells through a barrier membrane with or without particulate bone graft [4].

The issue is whether or not bone augmentation at the level of the implant remains stable on long-term when comparing with an implant placed in native bone. Only four studies compared the survival and success rates between implants with fenestration or dehiscence placed with the regenerated bone (test) and Implants placed in pristine bone [5-8]. These studies concluded that there was no significant difference in survival rates between the two groups Benic et al. [5,8] evaluated two groups in two different publications at five years and fifteen years interval. The marginal bone loss in 15 years amounted $1.44 \pm 0.84 \mathrm{~mm}$ for the GBR group and 1.69 $\pm 0.84 \mathrm{~mm}$ for the control group. From the 5 to the 15 years examination, the change in mean marginal bone loss reached $0.23 \pm 0.70 \mathrm{~mm}$ for the GBR group and $0.28 \pm 0.63 \mathrm{~mm}$ for the control. The survival rate for the implants combined with GBR-procedure and implants inserted in native bone amounted to $95.6 \%$ and $94.1 \%$ respectively after fifteen years [8]. This was in accordance with the two systematic reviews conducted separately by Sanz and Donos to evaluate the effectiveness of lateral bone augmentation on the level of the alveolar crest $[9,10]$. The implant survival at the augmented sites irrespective of the procedure used varied from $91.7 \%$ to $100 \%$ and from $93.2 \%$ to $100 \%$ at the sites where the implant was installed in native bone for a period between 12 and 59.1 months [10]. Chiapas found a survival rate of 95\% (range: $84 \%$ to $100 \%$ ) for the implants irrespective to the type of grafting material and membranes [11]. Considering these reports, one can conclude that the survival rate of implants in conjunction with GBR-procedure is equivalent to implants placed in native bone.

Despite the high survival rate of the dental implants in combination with GBR in the long-term, its assessment does not exactly provide all the information about implant health 
during the follow-up period. Peri-implantitis is defined as a plaque-associated pathological condition occurring in the tissues around dental implants and is characterized by inflammation in the peri-implant mucosa and subsequent progressive bone loss [12]. Peri-implant mucositis is described as bleeding on gentle probing around the implants whereas peri-implantitis is an increase in probing depth in addition to radiographic bone loss. Numerous long-term clinical studies and systematic reviews were conducted since the development of implant dentistry, to evaluate the biological alterations around the dental implants as well as its association with peri-implantitis. A recent systematic review reported two studies that showed the higher risk of periimplant mucositis and peri-implantitis in the simultaneous approach of implant placement with GBR when a residual defect is present at the time of re-entry [13].

In the present study, bone augmentation by guided bone regeneration in combination with bovine hydroxyapatite (Endobon ${ }^{\circledR}$ ) as filling material was evaluated with regard to hard tissue stability and peri-implant health.

\section{Materials and Methods}

Patient charts of 41 consecutively treated patients who had received bovine particulate bone augmentation Endobon ${ }^{\circledR}$ (Biomet 3i) were evaluated in a single center. From this cohort, 13 patients ( 3 female, 10 male) were enrolled in the study and the others were drop-outs due to the lack of radiographic material at the moment of implant placement. The mean value of the age was 50.5 (SD: 13.5; range 28-73).

17 implants were installed in the patients. All patients required horizontal ridge augmentation in order to install implants at the moment of implantation.

The approval of the ethical committee of the university hospital of Ghent was received prior to recruitment. Patient's age, gender, a region of implantation, type of implant, amount of graft material as well as the region of bone graft was recorded in patient's charts at the moment of implant placement. Patients were treated by clinical trainees in periodontology department of university hospital Gent. An intraoral radiograph was taken with a photostimulable phosphor plate after surgery and after loading by means of the long-cone parallel technique [14]. The patients were contacted 5 years after implant loading. All patients had undergone periapical radiographs and orthopantogram immediately after implant installation, after crown installation T0 and 5 years after implant loading T1. Digital and analog radiographs were evaluated from the patient charts after the crown installation. In three cases the referred dentists were asked to send the digital radiographs taken after installation of the crowns.

\section{Bone Level Measurements}

Radiographic measurements were performed at T0 with the implant-abutment platform as reference point by undergraduate master student and a trainee student in periodontology department. If the implant was placed sub crestal the measurement was from the top of the bone crest to the implant platform mesial and distal. At the second time point, the bone level was measured from the bone crest to the first bone to implant contact.

The radiographic picture was magnified and the level of crestal bone was assessed and measured at the mesial and distal margins of the implant. The bone level changes were calculated both mesially and distally, based on the baseline and the follow-up measurements. The difference between the two-time points on each implant sites showed the amount of bone loss.

\section{Clinical examination}

At the same appointment, a periodontal chart was taken, pocket depth, bleeding on probing and plaque amount was scrutinized around each implant.

Bleeding was assessed when there was actual bleeding after 30 seconds followed by probing. Pocket depth was measured at four sites from the marginal level of the gingiva to the deepest point of the probable pocket. The plaque was detected by the probe at the implant level. Peri-implant mucositis was defined as the presence of bleeding on probing without bone loss exceeding the normal bone remodeling [14]. All measurements were carried out using a periodontal probe $(\mathrm{CP}$ 12, Hu-Friedy Chicago, IL, USA)

\section{Statistical analysis}

The data were analyzed using the statistical package for the social sciences (SPSS) software. The mean average of the mesial and distal measurements of each implant at each period of time was calculated and noted an average bone loss each implant. The average of pocket depths was taken at each implant site. Descriptive statistics was conducted on all the variables. To compare mesial and distal measurements as well as evolutions, the Wilcoxon signed rank test was used. Detectable bone loss was measured from 0.5 (Standard deviation of radiographic bone loss).

\section{Results}

All implants survived after 5 years of loading. Statistically, significant bone loss was revealed between the baseline and the follow-up time at the implant sites. The overall mean marginal bone loss at the implant site was 0.69 (SD: 0.73). On the mesial sites for all but two implants $(0.03$ and 0.04$)$ the evolution was toward bone loss. On the distal sites for all but three implants $(0.01,0.02,0.02)$ the evolution was toward loss of bone. No difference in the bone loss was seen between mesial and distal sites. Figure 1 represents the frequency of bone loss between samples.

The mean pocket depth was 3.6 (SD: 1.03). The mean pocket depth measured separately at the buccal area was 3.04 (SD: 0.6).

$87.5 \%$ of the implant sites with bone augmentation showed bleeding on probing. The plaque was seen in $31.3 \%$ of the implant sites. Table 1 shows an overview of the bone loss and BOP around the implants. 


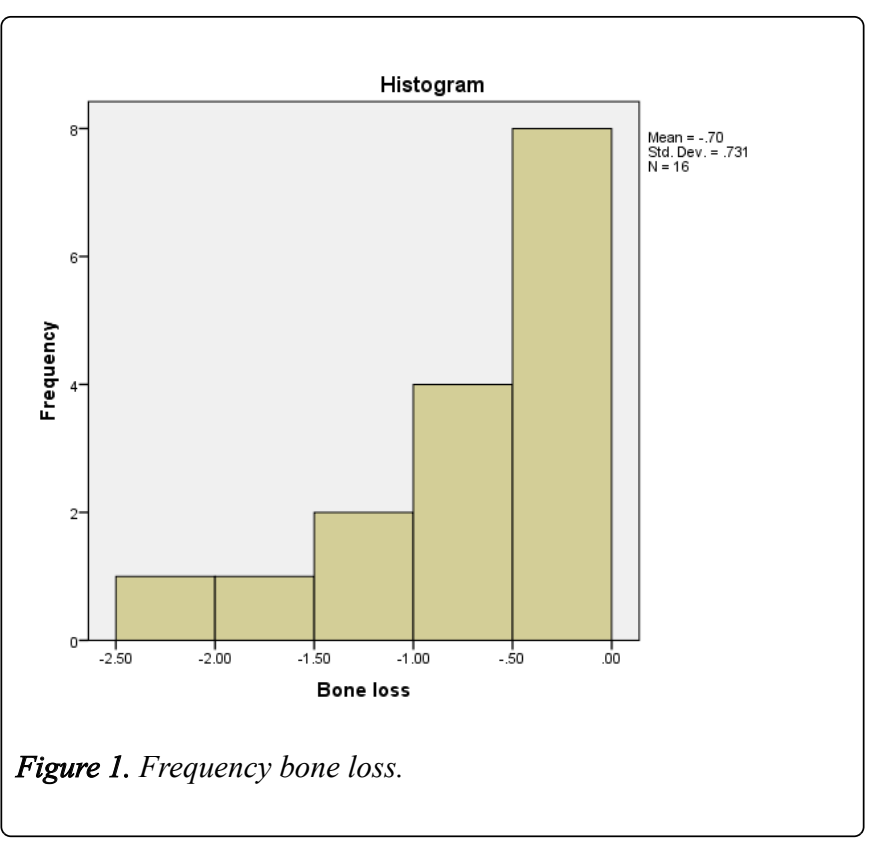

Table 1. Descriptive data analysis; Value in parentheses represents standard error for the value Marginal Bone Loss (MBL).

\begin{tabular}{|l|l|l|l|}
\hline variable & $\mathbf{5}$ y Follow-up & No & p-value \\
\hline MBL (mm) & $0.69(0.18)$ & 17 & 0.0005 \\
\hline Survival (\%) & 100 & 17 & n.s. \\
\hline
\end{tabular}

\section{Discussion}

Treatment of edentulous areas with a dental implant has been integrated into oral and dental surgery in the last decennia. The focus has been held to the treatment of more complex cases. The result of the present study showed that horizontal and vertical defects around the implants could be successfully treated with the GBR technique with the minimum remodeling over time.

The efficacy of different grafting materials has been widely reported in several studies. The Bovine Hydroxy Apatite (BHA) is considered a slow resorbing bone substitute that has osteoconductive properties and can be used as a space-filling material. In the present study, the bovine hydroxy apatite (Endobon ${ }^{\circledR}$ ) has been used as the space filling material. The slow resorption rate of the material has led to a successful and stable long-term stabilization around the implants.

In the present study, the implants were minimally rough implants (Biomet 3i, Palm Beach, FL, USA). A study evaluating Osseotite Biomet $3 \mathrm{i}$ implants for 10 years showed a mean marginal bone loss of $(0.76 \pm 0.18) \mathrm{mm}$ from the moment of implant placement and one year after the loading. The implants were immediately loaded with a provisional crown so that the biological width re-establishment can occur during implant healing. The results of follow-up over five and ten years showed no statistically significant bone loss Schropp et al. [15]. investigated bone loss around the same implant designs by taking radiographs after implant placement, after installation of the crown, one and five years subsequent to crown installation. Implants were in this study placed by terms of two-stage surgery.

Table 2. Parameters around each implant are noted in the table. Note the dark painted rows are the implants with $P D>6 \mathrm{~mm}$ and the $M B L$ exceeding the standard error. These criteria are suggested to diagnose peri-implantitis. BOP: Bleeding On Probing; PDM: Pocket Depth Mesial; PDD: Pocket Depth Distal; $\triangle M B L$ : Mean Marginal Bone Loss.

\begin{tabular}{|c|c|c|c|c|}
\hline Implant $\mathbf{n}$ & BOP & PDM & PDD & MBL \\
\hline 1 & 1 & 3 & 4 & 0.09 \\
\hline 2 & 1 & 5 & 5 & 2.58 \\
\hline 3 & 1 & 6 & 8 & 1.43 \\
\hline 4 & 1 & 5 & 3 & 1.13 \\
\hline 5 & 1 & 7 & 7 & 2.43 \\
\hline 6 & 1 & 5 & 5 & 0.08 \\
\hline 7 & 1 & 8 & 7 & 0.71 \\
\hline 8 & 1 & 3 & 3 & 0.62 \\
\hline 9 & 1 & 4 & 5 & 0.68 \\
\hline 10 & 1 & 3 & 4 & 0.12 \\
\hline 11 & 1 & 4 & 3 & 0.48 \\
\hline 12 & 0 & 3 & 5 & 0.9 \\
\hline 13 & 1 & 3 & 3 & 0.37 \\
\hline 14 & 0 & 5 & 3 & 0.07 \\
\hline 15 & 1 & 5 & 4 & 0.19 \\
\hline 16 & 1 & 2 & 3 & 0.09 \\
\hline 17 & 1 & 4 & 3 & 0.03 \\
\hline
\end{tabular}

Second stage surgery, for healings abutment placement, was performed three months after implant installation and the crown was placed 4 to 6 weeks afterward. A Significant bone loss of an average of $0.66 \mathrm{~mm}$ was found in all groups between implant placement and crown installation and an average bone loss of $0.54 \mathrm{~mm}$ between implant placement and one-year time point. No significant bone level changes were proved between the first year and ten years follow-up [16]. This data is comparable with the results of our study which shows a mean bone loss of $0.69 \mathrm{~mm}$. From these two last studies, we can conclude that on average, peri-implant bone adjusts rapidly during the first year after implant surgery mostly as a result of soft tissue adjustment. Initial crestal bone remodeling is highly affected by the biological width establishment which is up to $3 \mathrm{~mm}$ to $4 \mathrm{~mm}$ in the vertical dimension. Therefore, it is important to take the soft tissue vertical dimension in to account at the moment of implant placement when conducting a study that measures the crestal bone changes. Gilbert et al. [16], demonstrates a marginal bone level change of $0.35 \mathrm{~mm}$ around the moderately rough implants vs hybrid implants after a one-year follow-up in a split-mouth study. It is important to note that in this prospective study, the implants were placed with respect to the future biological width establishment. 
Nevertheless, Bone loss occurs after the first year, but the amount of bone loss is not significant unless there is a disbalance in the body's reaction to the implant. The latter can be a result of biological disbalance in the human body such as the history of periodontitis, smoking, and diabetes. Other factors are related to the surgical procedure, material, surface and implant design, prosthodontic components, clinician experience of the surgeon and prosthodontist and site related factors such as bone quality and quantity $[17,18]$.

In the present study, the amount of bone loss was 0.69 which was statistically significant. Given the fact that the biological width forming was not taken into account at the moment of implant insertion, this data is undisputed and is a matter of discussion which can be explained by reestablishment of biological width, prosthetic factors and the vertical position of the implants; though can be considered clinically insignificant. With other words, the bone loss could be just a normal biological reaction which is scientifically proven.

Three implants had pockets $>6 \mathrm{~mm}$ in this study and the mean bone loss measured from the baseline to the control was $2.43 \mathrm{~mm}, 1.13 \mathrm{~mm}$ and $1.43 \mathrm{~mm}$ (Table 2).

According to the 11th European consensus of Periodontology, the error of measurement should be taken as a threshold for bone loss. However, the study population of our study is small. Hence, the error of measurement is not reliable to diagnose peri-implantitis. The clinical diagnosis of periimplantitis is suggested to be based on a combination of pocket $>6 \mathrm{~mm}$ and bone level $>3 \mathrm{~mm}$ apical of the most coronal portion of the intraosseous implant [14]. Radiographical assessment of one patient out of three showed a gap in implant abutment junction that was a result of a bad fit of the supra construction. The excessive bone loss was most likely due to a prosthodontic complication (Figure 2). Yet, the bone level was not exceeding $3 \mathrm{~mm}$ apically of the most coronal portion; hence it is not diagnosed as periimplantitis.

In two patients the implants were installed $>2 \mathrm{~mm}$ subcrestal and the patients had a history of periodontitis but were treated at the moment of implantation. The results of a retrospective study revealed that sub crestal position of the implant $>2 \mathrm{~mm}$ results in higher marginal bone loss Negri et al. [19]. evaluated the influence of biological width formation in implants inserted at crestal bone level versus implants inserted $2 \mathrm{~mm}$ sub-crestal. Biological width dimensions were comparable in the different groups, ranging from $3.13 \pm 0.55$ $\mathrm{mm}$ to $3.34 \pm 0.53 \mathrm{~mm}$. In contrast, crestal bone loss was higher in the sub-crestal placed implants. Although sub crestal placement might reduce the distance from the implant shoulder to the first bone-to-implant contact, it might not have any minimizing effects on marginal bone loss. In this study, sub crestal placement of more than $2 \mathrm{~mm}$ resulted in higher crestal bone loss which was represented in two cases [20].

$84 \%$ of the patients showed peri-implant mucositis while healthy peri-implant conditions were seen in two patients in our study when applying the definition of European consensus [14]. Nevertheless, none of the implants revealed any signs of swelling, redness or purulence. Unfortunately, the lack of information about pocket depth at the baseline did not allow for any evaluation of pocket depth changes. Coli et al. [20] concluded in their review article that bleeding on probing and probing depth are not reliable tools for monitoring periimplant health or disease [21], namely: peri-implant probing can cause unnecessary trauma to the tissue [22]. Hence, the peri-implant disease must be diagnosed with caution and rapidly speaking of it can misguide us to overdiagnosis.

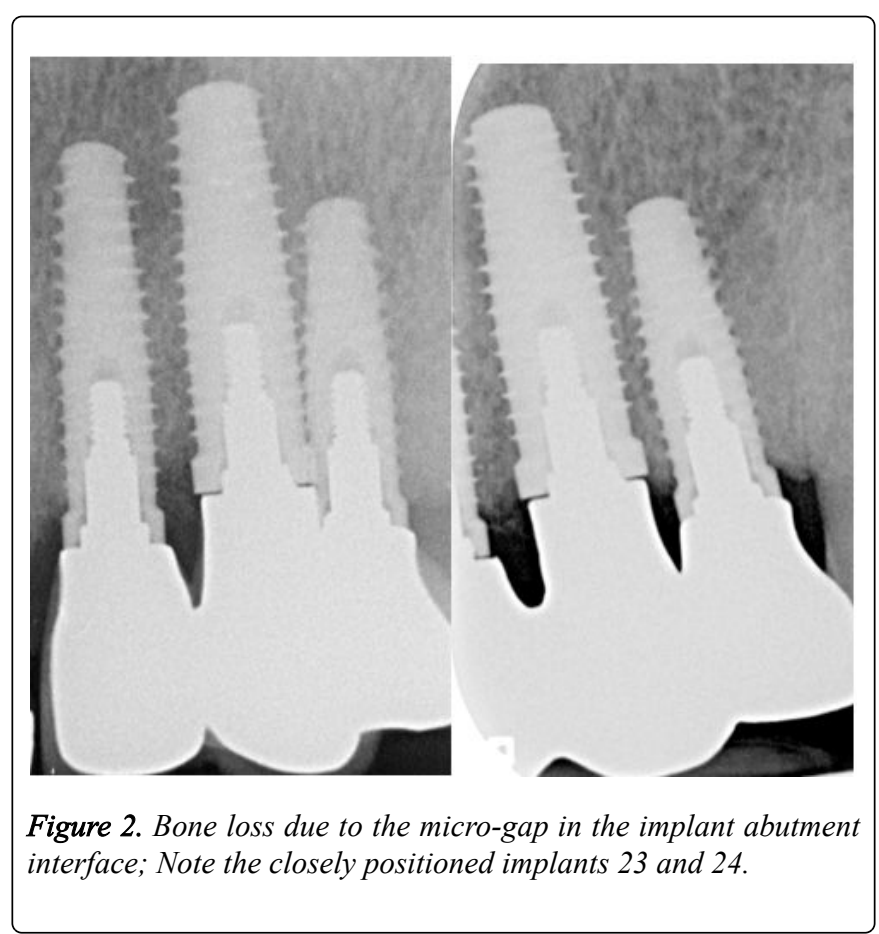

Schwarz et al. [22] compared the peri-implant condition of implants with no residual defects to implants with residual defect height of $<1 \mathrm{~mm}$ without bone augmentation and the implants with residual defects $>1 \mathrm{~mm}$ that were previously augmented with xenogeneic bovine bone. It was remarkable that the group with no GBR and no residual defects had significantly less bleeding on probing than the group with residual defect height of $<1 \mathrm{~mm}(29 \%)$ and with GBR (54\%). The results of his study revealed that the implants with residual defects higher than $1 \mathrm{~mm}$ that were treated with GBR are at higher risk for peri-implant disease. Therefore, the author suggested that GBR must be taken as a risk factor for peri-implant disease [23].

In our study, $87 \%$ of the buccal sites of the implants showed bleeding after gentle probing, which are the signs for mucositis. One hypothesis might be the stability of augmented bone at the moment of surgery. During the GBR procedure, the membrane that covers the bone particles is not always properly stabilized. If the wound has not reached the primary closure, the bone particles might release from the incision region and uncover the implant neck which in addition can cause a residual defect. As a consequence, this might be the reason for the high percentage of bleeding in our study. Most of the studies evaluating the bone level changes after GBR procedure use two-dimensional radiographs that only display mesial and distal bone level changes. Instead, bone augmentation is mainly used in the buccal area.

Three-dimensional tomography is an alternative to assess the accurate bone level in the buccal area [24]. So far, there are no studies comparing bone changes buccally on the basis 
of cone-beam tomography. This might be due to the radiation amount of CBCT that has no advantage for the patient in terms of treatment. Additionally, the scattering might not allow accurate measurement around the implants. Another alternative to assess accurate buccal bone level changes is bone sounding. Christiaens et al. [25] concluded that bone sounding without flap elevation is the most accurate measurement of peri-implant bone level taking the intra surgical bone level as a reference of true bone level This could be a future perspective for further research concerning bone level changes in long-term, subsequent to buccal bone augmentation.

Due to the retrospective nature of this study, there was some limitation primarily; the lack of site-level analysis at the moment of surgery and during the analysis did not allow us any evaluation of pocket depth changes and changes in recession or bleeding on probing.

\section{Conclusion}

Within the limitations of this study, we can conclude that bone augmentation with bovine bone hydroxyapatite in one stage implant surgery remained stable during the 5 years follow-up around the minimally rough implants used in this study. However, the amount of bone loss and mucositis at the implant sites with GBR remained significantly high.

\section{References}

1. Van der Weijden F, Dell'Acqua F, Slot DE. Alveolar bone dimensional changes of post-extraction sockets in humans: A systematic review. Journal of Clinical Periodontology. 2009; 36: 1048-1058.

2. Araújo MG, Lindhe J. Dimensional ridge alterations following tooth extraction. An experimental study in the dog. Journal of Clinical Periodontology. 2005; 32: 212-218.

3. Nyman S, Gottlow J, Lindhe J, Karring T, Wennstrom J. New attachment formation by guided tissue regeneration. Journal of Periodontal Research. 1987; 22: 252-254.

4. Liu J, Kerns DG. Mechanisms of guided bone regeneration: A review. The Open Dentistry Journal. 2014; 8: 56-65.

5. Benić GI, Jung RE, Siegenthaler DW, Hämmerle CHF. Clinical and radiographic comparison of implants in regenerated or native bone: 5-year results. Clinical Oral Implants Research. 2009; 20: $507-513$.

6. Mayfield L, Skoglund A, Nobréus N, Attström R. Clinical and radiographic evaluation, following delivery of fixed reconstructions, at GBR treated titanium fixtures. Clinical Oral Implants Research. 1998; 9: 292-302.

7. Aloy-Prosper A, Penarrocha-Oltra D, Penarrocha-Diago M, Penarrocha-Diago M. Dental implants with versus without periimplant bone defects treated with guided bone regeneration. Journal of Clinical and Experimental Dentistry. 2015: 361-368.

8. Benic GI, Bernasconi M, Jung RE, Hämmerle CHF. Clinical and radiographic intra-subject comparison of implants placed with or without guided bone regeneration: 15-year results. Journal of Clinical Periodontology.2017; 44: 315-325.

9. Sanz-Sánchez I, Ortiz-Vigón A, Sanz-Martín I, Figuero E, Sanz M. Effectiveness of lateral bone augmentation on the alveolar crest dimension: A systematic review and meta-analysis. Journal of Dental Research. 2015; 94: 128S-142S.

10. Donos N, Mardas N, Chadha V. Clinical outcomes of implants following lateral bone augmentation: systematic assessment of available options (barrier membranes, bone grafts, split osteotomy). Journal of Clinical Periodontology. 2008; 35: 173-202.
11. Chiapasco M, Zaniboni M. Clinical outcomes of GBR procedures to correct peri-implant dehiscences and fenestrations: A systematic review. Clinical Oral Implants Research. 2009; 20: 113-123.

12. Lindhe J, Meyle J. Group D of European workshop on periodontology. Peri-implant diseases: Consensus report of the sixth European workshop on periodontology. Journal of Clinical Periodontology. 2008; 35: 282-285.

13. Berglundh T, Armitage G, Araujo MG, Avila-Ortiz G, Blanco J, et al. Peri-implant diseases and conditions: Consensus report of workgroup 4 of the 2017 World Workshop on the classification of periodontal and peri-implant diseases and conditions. Journal of Clinical Periodontology. 2018; 45: S286S291.

14. Calvo-Guirado JL, Gómez-Moreno G, Delgado-Ruiz RA, Maté Sánchez de Val JE, Negri B, et al. Clinical and radiographic evaluation of osseotite-expanded platform implants related to crestal bone loss: A 10-year study. Clinical Oral Implants Research. 2014; 25: 352-358.

15. Schropp L, Wenzel A, Stavropoulos A. Early, delayed, or late single implant placement: 10-year results from a randomized controlled clinical trial. Clinical Oral Implants Research. 2014; 25 : 1359-1365.

16. Glibert M, Matthys C, Maat RJ, De Bruyn H, Vervaeke S. A randomized controlled clinical trial assessing initial crestal bone remodeling of implants with different surface roughness. Clinical Implant Dentistry and Related Research. 2018; 20: 824-828.

17. De Bruyn H, Christiaens V, Doornewaard R, Jacobsson M, Cosyn J, et al. Implant surface roughness and patient factors on longterm peri-implant bone loss. Periodontology 2000. 2017; 73: 218-227.

18. Madani E, Smeets R, Freiwald E, Sanj MS, Jung O, et al. Impact of different placement depths on the crestal bone level of immediate versus delayed placed platform-switched implants. Journal of Cranio-Maxillofacial Surgery. 2018; 46: 1139-1146.

19. Negri B, López Marí M, Maté Sánchez de Val JE, Iezzi G, Bravo González LA, et al. Biological width formation to immediate implants placed at different level in relation to the crestal bone: An experimental study in dogs. Clinical Oral Implants Research. 2015; 26: $788-798$.

20. Coli P, Christiaens V, Sennerby L, Bruyn HD. Reliability of periodontal diagnostic tools for monitoring peri-implant health and disease. Periodontology 2000. 2017; 73: 203-217.

21. Albrektsson T, Chrcanovic B, Östman PO, Sennerby L. Initial and long-term crestal bone responses to modern dental implants. Periodontology 2000. 2017; 73: 41-50.

22. Schwarz F, Sahm N, Becker J. Impact of the outcome of guided bone regeneration in dehiscence-type defects on the longterm stability of peri-implant health: clinical observations at 4 years: Long-term stability of GBR. Clinical Oral Implants Research. 2012; 23: 191-196.

23. Timock AM, Cook V, McDonald T, Leo MC, Crowe J, et al. Accuracy and reliability of buccal bone height and thickness measurements from cone-beam computed tomography imaging. American Journal of Orthodontics and Dentofacial Orthopedics. 2011; 140: 734-744.

24. Corpas L dos S, Jacobs R, Quirynen M, Huang Y, Naert I, et al. Peri-implant bone tissue assessment by comparing the outcome of intra-oral radiograph and cone beam computed tomography analyses to the histological standard. Clinical Oral Implants Research. 2011; 22: 492-499.

25. Christiaens V, Jacobs R, Dierens M, Vervaeke S, De HB, et al. Intraoral radiography lacks accuracy for the assessment of periimplant bone level-a controlled clinical study. European Journal of Oral Implantology. 2017; 10: 435-441. 